\title{
Impactos ambientais de metais pesados de pilhas na tríade água-ar-solo
}

Environmental impacts of heavy metals from batteries in the water-air-soil triad

\section{IsabellySette Barroco}

Grupo Interdisciplinar de Educação, Eletroquímica, Saúde, Ambiente e Arte (GIEESAA), Universidade Federal do Rio de Janeiro (UFRJ), DFQ, Instituto de Química.

isabellysette@gmail_com orcid.org/0000-0002-1814-8308

\section{Fernanda dos Santos Castro}

Grupo Interdisciplinar de Educação, Eletroquímica, Saúde, Ambiente e Arte (GIEESAA), Universidade Federal do Rio de Janeiro (UFRJ), DFQ, Instituto de Química. nanda.castro97s@gmail.com orcid.org/0000-0001-9762-5928

\section{Priscila Tamiasso-Martinhon}

Grupo Interdisciplinar de Educação, Eletroquímica, Saúde, Ambiente e Arte (GIEESAA), Universidade Federal do Rio de Janeiro (UFRJ), DFQ, Instituto de Química. Núcleo de Estudos em Biomassa e Gerenciamento de Águas (NAB), UFF. pris-martinhon@hotmail.com orcid.org/0000-0001-6141-3755

\section{Angela Sanches Rocha}

Grupo Interdisciplinar de Educação, Eletroquímica, Saúde, Ambiente e Arte (GIEESAA), Núcleo de Estudos em Biomassa e Gerenciamento de Águas (NAB), UFF, Universidade do Estado do Rio de Janeiro (UERJ), DFQ, Instituto de Química. 


\section{Celia Regina Sousa}

Grupo Interdisciplinar de Educação, Eletroquímica, Saúde, Ambiente e Arte (GIEESAA), Universidade Federal do Rio de Janeiro (UFRJ), DFQ, Instituto de Química. Núcleo de Estudos em Biomassa e Gerenciamento de Águas (NAB), UFF. sousa@iq.ufrj.br orcid.org/0000-0001-8988-3724

Resumo. Com o aumento da fabricação de produtos tecnológicos, faz-se necessário a utilização de fontes de energia portáteis, como pilhas. Sua utilização e descarte quando feitos de forma consciente conferem às pilhas um status de grande importância no dia a dia da sociedade. Contudo, quando o descarte é realizado de forma incorreta, seus efeitos negativos são evidenciados. Em vista disso, este trabalho almeja, por meio de uma revisão bibliográfica, investigar os impactos ambientais causados pelos metais pesados presentes em pilhas domésticas, quando estas são descartadas de forma inadequada. Além disso, são discutidos aspectos relativos aos principais tipos de pilhas presentes no mercado, como também a aplicabilidade da legislação que regula o descarte de resíduos tóxicos.

Palavras-chave: Metais pesados. Impactos ambientais. Pilhas

Abstract.With the increase in the manufacture of technological products, it is necessary to use portable energy sources, such as batteries. Its use and disposal when done consciously give batteries a status of great importance in society's daily life. However, when the disposal is performed incorrectly, its negative effects are evident. In view of this, this work, through a bibliographic review, investigates the environmental impacts caused by the heavy metals present in the batteries, when they are disposed of inappropriately. In addition, aspects related to the main types of batteries on the market are discussed, as well as the application of legislation that regulates or discards toxic waste.

Keywords: Heavy metals. Environmental impacts. Batteries

Recebido: 01/10/2018 Aceito: 27/10/2018 Publicado: 05/11/2018

\section{Introdução}

Hoje, no mundo globalizado em que vivemos, há a necessidade constante de se encurtarem as distâncias entre as pessoas, favorecendo assim um grande fluxo de informações e relações interpessoais (BARBOSA, 2001). Como forma de se adaptar a essa nova conjuntura e suprir as necessidades do consumidor, a indústria tecnológica 
desenvolve diariamente novos produtos eletroeletrônicos como celulares, computadores, televisores, rádios, entre outros, para que estes atendam a demanda atual (SENA, 2012). Contudo, devido ao dinamismo das relações de mercado, grande parte destes produtos apresentam vida útil curta ou são rapidamente substituídos por tecnologias mais atuais, evidenciando então a necessidade do debate a respeito da forma correta de descartá-los.

Grande parte dos equipamentos eletrônicos portáteis ou utilizados em casa necessitam, para seu funcionamento, de fontes energéticas como pilhas. No Brasil, de acordo com os dados da Associação Brasileira da Indústria Elétrica e Eletrônica (ABINEE) são comercializados ao ano em torno de 1,2 bilhões de pilhas e baterias. No entanto, somente uma pequena parcela é descartada de forma correta e reciclada, já a grande maioria deste resíduo sólido é eliminado no lixo comum, tendo como destino lixões e aterros sanitários, o que se constitui como um grande problema ambiental (KEMERICH et al., 2012).

As pilhas podem parecer inofensivas, porém são consideradas lixos tóxicos, pois contêm metais pesados em sua composição como $\mathrm{Hg}, \mathrm{Cd}, \mathrm{Mn}, \mathrm{Zn}, \mathrm{Pb}, \mathrm{Ni}$, dentre outros. Quando descartadas de forma irregular, causam grandes impactos ambientais e socioambientais, ocasionados pelo processo de bioacumulação de metais pesados (AFONSO et al., 2003). Sendo assim, torna-se necessário o estímulo emergencial da reciclagem desses resíduos. Contudo, essa medida esbarra em uma série de empecilhos, entre eles a pequena quantidade de postos de coletas de lixos tóxicos e, principalmente, a baixa viabilidade econômica do processo, o que demanda altos custos para as empresas produtoras de resíduos.

Apesar de ser considerado o melhor método para reduzir o impacto ambiental, a reciclagem é pouco discutida no Brasil e também carece de pesquisas voltadas para a recuperação de resíduos metálicos oriundos de pilhas. Dessa forma, o escopo desse artigo é direcionado para a análise dos problemas ambientais causados pela ação de metais pesados.

\section{Metodologia}

Como fundamentação teórica para a pesquisa foram realizadas buscas em plataformas da internet e, principalmente, nas bases de dados Web of Science e Scielo, para serem obtidas informações a respeito de como está ocorrendo o desenvolvimento e descobertas relativas ao referido campo de pesquisa.

Por se tratar de um tema multidisciplinar, a revisão sistemática da literatura foi pautada em um levantamento de artigos e teses relativos aos impactos decorrentes de resíduos tóxicos, como os ocasionados pelo descarte de pilhas. Dessa forma, as buscas foram baseadas na avaliação dos impactos causado no meio ambiente, levando em consideração seus efeitos sobre os elementos da natureza como água, ar e solo. 


\section{Resultados e discussões}

A partir da análise da bibliografia selecionada, mediante ao estabelecimento de critérios para as buscas, foi possível examinar os impactos ambientais decorrentes de resíduos metálicos de pilhas reportados.

Dentre os 118 elementos químicos, 84 são metais (IUPAC) sendo alguns destes fundamentais para a manutenção do equilíbrio do ecossistema e sua ocorrência, quando de forma natural, não é configurada como perigosa para o ambiente. Contudo, devido à ação do homem, houve um aumento na concentração de metais no meio ambiente.

A contaminação ambiental por metais pesados é um fator que contribui diretamente para a poluição do ar, dos solos e sistemas aquáticos, provocando a mortandade de espécies, assim como intoxicação do homem. Grande quantidade desses metais que se depositam no ambiente sem qualquer tipo de tratamento são decorrentes de atividades antrópicas. Com base nesta informação, tornou-se necessário o aumento no número de palavras pesquisadas, a fim de avaliar tais impactos no meio ambiente, sendo estes muitas vezes irreversíveis.

Dentre os diversos fatores que contribuem para a poluição do meio ambiente por metais, destaca-se a disposição de pilhas no lixo comum. No Brasil, de acordo com os dados da Associação Brasileira da Indústria Elétrica e Eletrônica (ABINNE), as principais pilhas utilizadas são as de zinco-carbono e alcalinas, ambas constituídas em sua maioria por zinco e manganês, sendo sua diferença principal na constituição do eletrólito, a primeira composta por óxido de manganês e a segunda por hidróxido de potássio. Em relação aos valores de toxidade, dentre as principais marcadas comercializadas, ambas respeitam os parâmetros definidos pelas resoluções do Conselho Nacional do Meio Ambiente (CONAMA): $n^{o}$ 401, de 04/11/2008; e no 424, de 22/04/2010. Contudo, apesar da legislação atribuir a responsabilidade da destinação adequada de rejeitos tóxicos aos fabricantes e importadores, o problema não é solucionado de fato devido à falta de informação do consumidor, assim como a pequena quantidade de processos eficientes de tratamento de rejeitos de pilhas. Portanto, a destinação final desses rejeitos tóxicos, muitas vezes, acaba sendo realmente o lixo domiciliar.

Ademais, para se ter um entendimento em relação ao panorama geral, é necessário avaliar os diversos tipos de pilhas disponíveis no mercado. As pilhas podem ser divididas em dois grupos, sendo eles de baterias primárias (pilhas descartáveis) e baterias secundárias (recarregáveis), sendo as primeiras grandes responsáveis pelos maiores impactos ambientais devido a sua pequena vida útil. A Tabela 1 apresenta uma relação das características das principais pilhas comercializadas, tornando possível a identificação dos metais presentes nelas.

Tabela 1. Tipos de pilhas disponíveis no mercado. 


\begin{tabular}{|c|c|c|c|c|c|}
\hline Classificação & Bateria & Anodo & Catodo & Eletrólito & $\begin{array}{l}\text { Regulamen } \\
\text {-tação }\end{array}$ \\
\hline \multicolumn{6}{|l|}{$\mathrm{P}$} \\
\hline $\begin{array}{l}\mathrm{R} \\
\mathrm{I}\end{array}$ & $\begin{array}{l}\text { Zinco- } \\
\text { Carbono }\end{array}$ & $\mathrm{Zn}$ & $\mathrm{MnO}_{2}$ & $\mathrm{NH}_{4} \mathrm{Cl}$ & Sim \\
\hline M & Alcalina & Zn (pó) & $\mathrm{MnO}_{2}$ & $\mathrm{KOH}$ & Sim \\
\hline Á & $\mathrm{Zn}-\mathrm{Ag}_{2} \mathrm{O}$ & $\mathrm{Zn}$ & $\mathrm{Ag}_{2} \mathrm{O}$ & $\mathrm{KOH}$ & Não \\
\hline $\begin{array}{l}\mathrm{R} \\
\mathrm{I}\end{array}$ & Zinco-Ar & $\mathrm{Zn}$ & $\mathrm{O}_{2}$ & $\mathrm{KOH}$ & Não \\
\hline $\begin{array}{l}\mathrm{A} \\
\mathrm{S}\end{array}$ & $\mathrm{Li}-\mathrm{MnO}_{2}$ & $\mathrm{Li}$ & $\mathrm{MnO}_{2}$ & $\begin{array}{l}\text { Sal de Li não } \\
\text { aquoso }\end{array}$ & Não \\
\hline \multicolumn{6}{|l|}{$\mathrm{S}$} \\
\hline E & Pb-Ácido & $\mathrm{Pb}$ & $\mathrm{PbO}_{2}$ & $\mathrm{H}_{2} \mathrm{SO}_{4}$ & Sim \\
\hline $\begin{array}{l}\mathrm{C} \\
\mathrm{U}\end{array}$ & $\mathrm{Ni}-\mathrm{Cd}$ & $\mathrm{Cd}$ & $\mathrm{NiO}$ & $\mathrm{KOH}$ & Sim \\
\hline $\begin{array}{l}\mathrm{N} \\
\mathrm{D}\end{array}$ & Ni-MH & $\begin{array}{l}\mathrm{MH}(\mathrm{V}, \\
\mathrm{Ti}, \mathrm{Ni})\end{array}$ & $\mathrm{NiOOH}$ & $\mathrm{KOH}$ & Não \\
\hline $\begin{array}{l}\mathrm{A} \\
\mathrm{R} \\
\mathrm{I}\end{array}$ & Li-íon & Grafite & $\mathrm{LiCoO}_{2}$ & $\begin{array}{l}\mathrm{LiPF}_{6} \text { em } \\
\text { solvente } \\
\text { orgânico }\end{array}$ & Não \\
\hline $\begin{array}{l}\mathrm{A} \\
\mathrm{S}\end{array}$ & & & & & \\
\hline
\end{tabular}

Fonte: PAUL (2016), BOCCHI et al. (2000), CONAMA.

É possível verificar que somente determinados tipos de pilhas e baterias são regulamentados pela legislação vigente, sendo as demais passíveis de políticas mais complexas de coleta seletiva, o que se constitui como um elemento dificultador para seu descarte adequado. $\mathrm{O}$ processo de retorno desses rejeitos aos fabricantes para o tratamento adequado é complexidade, o que agrava o problema e propicia o descarte inadequado desses objetos, contribuindo assim para a poluição ambiental, no caso de rompimento do envoltório e vazamento da pasta eletrolítica no ambiente.

As grandes quantidades de metais expostos ao ambiente produzem impactos nos solos, sistemas aquáticos e ar por meio de fenômenos como lixiviação, troca química, sedimentação, adsorção, entre outros.

\subsection{Impactos no solo}


Quando o destino desses metais tóxicos sem tratamento é o solo, sua presença promove contaminação desse ambiente, constituindo assim uma 'área contaminada'. Área contaminada pode ser definida como sendo um local de potencial risco para a saúde humana e de prejuízo dos recursos ambientais em virtude da concentração de elementos tóxicos em sua composição. (CUNHA, 1997).

De acordo com os estudos relativos aos impactos nos solos decorrentes da exposição destes à metais pesados, foi verificado que os graus de mobilidade e biodisponibilidade dos metais dependem da composição do solo, sendo os teores de matéria orgânica e inorgânica significativos para as reações de precipitação (OLIVEIRA; MATTIAZZO, 2001) assim como aspectos como $\mathrm{pH}$, temperatura e competição com outros metais possuem influência (OLIVEIRA; COSTA, 2004).

Como exemplo disso, por meio de experimentos, foi verificado que a mobilidade de $\mathrm{Zn}$ e Mn de pilhas alcalinas e de zinco-carbono presentes é maior quando o $\mathrm{pH}$ do solo apresenta valores menores do que 5, indicando, portanto, a influência do $\mathrm{pH}$ na composição metálica do solo (AGOURAKIS et al., 2006). Ademais, outro aspecto que agrava o panorama é a migração compostos contaminantes. Estes podem migrar e se concentrar nas plantas e animais, por meio da utilização de um solo contaminado para atividade de agricultura e pecuária, permitindo assim bioacumulação pela cadeia alimentar. Além disso, podem promover impactos nas comunidades microbianas e fúngicas do solo, como também podem migrar para aquíferos, agravando a agressão ambiental (GÜNTHER, 1998).

\subsection{Impactos nas águas superficiais e subterrâneas}

Outro aspecto relativo à contaminação ambiental está relacionado à poluição das águas por contaminantes metálicos. Os metais que existentes na natureza não se apresentam como vetores de riscos à saúde, contudo quando presentes em grandes concentrações devido a ações antrópicas, interagem com a água, contribuindo assim para a modificação de sua biodisponibilidade e grau de toxidade.

Quando os metais são introduzidos na água, seja de forma direta como por exemplo o descarte de pilhas em locais inadequados, ou por meio do carreamento de partículas metálicas proveniente de solos contaminados, estes são adsorvidos na superfície de componentes em suspensão na água e coexistem com a fase metálica dissolvida na água. Dentre os aspectos que se constituem como fundamentais para a contaminação de ambientes aquáticos se destacam as interações entre os metais introduzidos nas águas e os constituintes orgânicos e inorgânicos anteriormente presentes, além de aspectos como $\mathrm{pH}$ e o grau de solubilidade do metal na área afetada (GÜNTHER, 1998). Sob determinadas condições, podem ter transformados em íons livres a partir de sua forma molecular elementar (GÜNTHER, 1998). Em relação ao mercúrio, apesar deste representar uma pequena parcela da massa metálica total da qual uma pilha é 
constituída, sua exposição ao meio ambiente e sua ocorrência nas águas representam fatores de risco para as comunidades aquáticas da região. O mercúrio pode ser transformado em um composto muito mais tóxico, $\left(\mathrm{CH}_{3}\right)_{2} \mathrm{Hg}$, que sua forma molecular elementar (Hg), o que agrava a situação, visto que é um composto com maior solubilidade e de mais fácil assimilação pelos organismos de diferentes espécies (GÜNTHER, 1998).

Uma das formas de analisar a contaminação das águas de determinada região está relacionada com a forma de toxicidade da água para a espécies aquáticas (BRANCO, 1960). Apesar de determinadas concentrações de metais não serem tóxicas para todas as espécies, o que culmina com uma quantidade de peixes resistentes à contaminação, seus impactos se estendem ao homem, visto que resultam no processo de bioacumulação ao longo da cadeia alimentar.

\subsection{Impactos no ar}

A disposição de pilhas de forma inadequada tem como resultado a exposição desses resíduos sólidos não somente a ação natural, como também a ação humana. Muitas vezes por falta de conhecimento dos malefícios dos componentes metálicos desse rejeito sólido, a adoção da prática de queima de resíduos contribui por agravar a questão da poluição do ar. Quando medidas que visam à eliminação ou à redução do lixo eletrônico são realizadas por meio da queima do material, estas acabam por liberar vapor tóxico, situação que resulta em aumentar a poluição, não só do ar, como também de outros meios naturais.

Quando partículas de metais pesados se encontram presentes na atmosfera em altas concentrações, devido ao ciclo hidrológico, acabam por culminar com a poluição difusa, definida por Günther como sendo: ' ...resultado das emissões atmosféricas que retornam a superfície do solo'". Portanto, é possível verificar que dentre os elementos nocivos presentes nas pilhas, com destaque para o cádmio, mercúrio e chumbo, o material particulado oriundo desses constituintes quando lançados na atmosfera resultam em vapores altamente tóxicos que expõe todo o equilíbrio do ecossistema a situações de risco.

\section{Conclusões}

Levando em consideração os aspectos relativos aos resultados encontrados, por meio da discussão apresentada, foi possível verificar que os impactos negativos resultantes de rejeitos metálicos oriundos de pilhas afetam todas as esferas naturais. A má utilização e seu descarte quando feito de forma incorreta ocasionam consequências ambientais e socioambientais graves e muitas vezes irreversíveis. No entanto, é valido destacar que os aspectos positivos da utilização de pilhas não podem ser desprezados. 
O presente trabalho não pretende apenas classificar as pilhas como vilãs e poluidoras do meio ambiente, mas a abordagem de pesquisa escolhida se limita aos impactos no meio ambiente, que não negativos. Porém compreendemos sua importância na vida moderna, sendo necessário uma reflexão sobre seu uso desenfreado e, principalmente seu descarte adequado.

Torna-se notório que os problemas apresentados são graves e que necessitam de medidas emergenciais para que possam ser minimizados e até mesmo solucionados. À vista disso, é necessário então que haja uma maior conscientização a respeito dos riscos de rejeitos sólidos considerados tóxicos, assim como ocorra um maior estímulo à reciclagem no Brasil. Para isso, como ponto principal, se faz necessário a discussão da legislação brasileira a respeito da regulamentação.

\section{Financiamento}

O presente trabalho foi realizado com apoio do Conselho Nacional de Desenvolvimento Científico e Tecnológico (CNPq)

\section{Referências}

ABINNE, Associação Brasileira da Indústria Elétrica e Eletrônica. A Indústria Elétrica e Eletrônica Impulsionando a Economia Verde e a Sustentabilidade. 2012.

AFOnSO, J. C.; BARANDAS, A. P. M. G.; SilvA, G. A. P. FOnSECA, S. G. Processamento da pasta eletrolítica de pilhas usadas.Química Nova, v. 26, n. 4. 2003.

AgOURAKIS, D. C.; CAMARGO, I. M. C.; COTRIM, M. B.; FlueS, M. Comportamento de Zinco e Manganês de Pilhas Alcalinas em uma Coluna de Solo.Química Nova, v. 29, n. 5, p.960-964, Set/Out, 2006.

BARBOSA, S. P. Impacto da Globalização sobre o Princípio da Eficiência.Revista de direito administrativo, n. 244. Rio de Janeiro: Editora Renovar. 2001.

BOCCHI, N.; FERRACIN, L. C.; BIAGGIO, S. R. Pilhas e Baterias: Funcionamento e Impacto Ambiental.Química Nova na Escola, n. 11, 2000. 
BRANCO, S. M. Observações sobre o Comportamento de Peixes em Presença de Certos Componentes Metálicos Dissolvidos na Água. Revista DAE, ed. 37, p. 37-41, 1960.

BRASIL. Ministério do Meio Ambiente (MMA). Conselho Nacional do Meio Ambiente (CONAMA). Resolução CONAMA n 401, de 04/11/2008: Diário Oficial da União em 05 de Novembro de 2008.

BRASIL. Ministério do Meio Ambiente (MMA). Conselho Nacional do Meio Ambiente (CONAMA). Resolução CONAMA n' 424, de 22/04/2010:Art. 16 da Resolução CONAMA no 401/2008. Diário Oficial da União em 23 de Abril de 2010.

CUNHA, R. C. A. Avaliação do Risco de Áreas Contaminadas por Fontes Industriais Desativadas - Estudo de Caso. Tese de Doutorado - Instituto de Geociências, Universidade de São Paulo. São Paulo, 1998.

GÜNTHER, W. M. R. Contaminação Ambiental por Disposição Inadequada de Resíduos Industriais Contendo Metais Pesados - Estudo de Caso. Tese de Doutorado - Faculdade de Saúde Pública, Universidade de São Paulo. São Paulo, 1998.

IUPAC, Internacional Union of Pure and Applied Chemistry. Discovery and Assignment of Elements with Atomic Numbers 113, 115, 117 and 118, 2015.

KEMERICH, P. D. C.; MENDES, S. A.; VORDAGEL, T. H.; PIOVESAN, M. Descarte Indevido de Pilhas e Baterias: A Percepção do Problema no Município de Frederico Westphalen - RS.Revista Eletrônica em Gestão, Educação e Tecnologia Ambiental, v. 8, n.8, p. 1860-1688, 2012.

OliveirA, F. C.; MATTIAZZO, M. E. Mobilidade de Metais Pesados em um Latossolo Amarelo Distrófico Tratado com Iodo de Esgoto e Cultivado com Cana- deAçúcar.Scientia Agrícola, v. 58, n. 4, p. 807-812, 2001. 
OLIVEIRA, T. S.; COSTA, L. M. Metais Pesados em Solos de uma Topolitossequência do Triângulo Mineiro.RevistaBrasileira de Ciências do Solo, v. 28, p. 785-796, 2004.

PAUL, S. Materials and Electrochemistry: Present and Future Battery. Journal of Electrochemical Science and Technology,p. 115-131, 2016.

SENA, F. R. Evolução da Tecnologia Móvel Celular e o Impacto nos Resíduos de Eletroeletrônicos. Dissertação de Mestrado - Departamento de Engenharia Civil, Pontifícia Universidade Católica do Rio de Janeiro. Rio de Janeiro, 2012. 